\title{
Cableless Measurement of Hand Griping Patterns and Heart Rate with Flexible Pressure Sensor Sheet
}

\author{
Tianyi Wang ${ }^{1}$, Hieyong Jeong ${ }^{1 *}$, An Guo ${ }^{1}$, Takafumi Ohno ${ }^{1}, \mathrm{Na} \mathrm{Li}^{1}$, and Yuko Ohno ${ }^{2}$ \\ ${ }^{1}$ Department of Robotics \& Design for Innovative Healthcare, Graduate School of Medicine, Osaka University, Osaka, \\ Japan \\ ${ }^{2}$ Department of Mathematical Health Science, Graduate School of Medicine, Osaka University, Osaka, Japan
}

\begin{abstract}
Background: Driver's behaviours such as poor steering patterns and drowsy driving at the steering wheel have been recognized as main causes of traffic accidents. However, little researches focused on how drivers hold the steering wheel and previous studies have not addressed cableless approach to measure heart rate from hand griping. In this paper, we proposed a basic study on measuring hand griping patterns and heart rate simultaneously through hand griping using flexible pressure sensor sheet.
\end{abstract}

Methods: Six volunteers were asked to steer a cylinder bar with six different hand griping patterns and steer still for 60 seconds for five times, hand griping pressure was measured using pressure sensor sheet, hand griping patterns were identified by hand griping pressure

Correspondence to:

Hieyong Jeong

Department of Robotics \& Design for Innovative Healthcare,

Graduate School of Medicine, Osaka University, Osaka, Japan.

E-mail: h.jeong@sahs.med.osaka-u.ac.jp distribution, heart rate was calculated from hand griping pressure filtered by band pass filter (BPF) and the results were crosschecked by gold standard measurement system.

Results: According to experiment results, proposed flexible pressure sensor sheet can clearly perform hand griping patterns, heart rate detection showed high accuracy (94\%) and good agreement compared with gold standard measurement system.

Conclusion: It was concluded that proposed method showed high potential application for measuring hand griping patterns and heart rate with cableless measurement.

\section{Keywords}

Medical informatics; Evaluation studies; Telemedicine; Patient participation; Patient portals

EJBI 2018; 14(4):04-11

Received: March 23, 2018

Accepted: May 04, 2018

Published: May 11, 2018

\section{Introduction}

The number of death due to traffic accidents in 2013 reached 1.25 million, summarized by World Health Organization [1], this report also pointed out the road traffic injuries leaded the main reasons of death in 2012 among young people aged 15-29 years, followed by suicide and AIDS/HIV.

Driver errors involving poor steering skills were recognized as the main causes of vehicle accidents [2]. Discussion about how drivers place their hands and change hand position usually used language of clocks. A '10 and 2 clock' steering technique is frequently advocated at driving schools in the UK and on the highway [3]. Now in the United States, ' 9 and 3 clock' is also optional even ' 8 and 4 clock' is accepted when driving for long distance on the freeway. It cannot be denied that some drivers ignored the strict traffic laws and regulations, which undoubtedly placed passengers and drivers themselves in high risk of car accident. For this reason, recent study tried to assess different steering techniques in order to characterize the drivers' steering profile by tracking hand position using simulators and motion capture system [4]. However, only focusing on hand position can hardly ensure maintenance of car control and react ability at any time. Topics on how drivers hold the steering wheel should be paid more attention, so that we can take a close look at drivers' performance. Unfortunately, understanding how human exploit hand function and sensory feedback of hand griping pattern is a challenging topic that still is not completely understood [5].

Besides inappropriate steering pattern behind the steering wheel, drowsy driving should take responsibility for $12.5 \%$ of vehicle crashes requiring medical treatment both for drivers and passengers, which were surveyed by The American Automobile Association [6]. This is not surprised because most commercial drivers especially truck drivers frequently drive on a long, monotonous and high-speed roadway at irregular time that may conflict with natural 
circadian rhythms, as a consequence they may have limited time to obtain sufficient sustained and restorative sleep [7]. As much as alcohol, drowsiness can impair driving performance including reaction time [8], ability to make judgment and process complex information on the road [9] and unable drivers uphold vigilance $[10,11]$.

As a consequence, great deals of researches have studied on this topic, Yu et al. [12] proposed a non-intrusive way to detect driver drowsiness, using two conductive fabrics on seat backrest to measure electrocardiograph (ECG) signal. Experiment results demonstrated the ECG signal was of satisfiable energy level and successfully showed driver's heart rate variability with drowsiness. However, such approach required electric nodes that may encumber driver's hands movements while maneuvering the vehicles and make drivers uncomfortable. Clapers et al. [13] proposed a wireless steering wheel in order to perform fast shortterm ECG acquisition and heart rate measurement. However, subjects have to place their hands at particular position, where their hands are connected with electrodes, in order to acquire the ECG signal. In real drive condition, drivers change their hands position all the time when changing the speed or turn the car, thus it is difficult to ensure drivers put their hands at the same position. Hand position-free method to measure drivers' biological signal is necessary.

Thus we thought it would be high value to apply cableless sensor technology to measure driver's hand griping patterns and heart rate simultaneously through hand griping. Figure 1 demonstrates the concept of our study. In this study, we aim to measure hand griping patterns and heart rate by using a flexible pressure sensor sheet, which can be fitted for hand griping. This paper is organized into four sections. Section 2 explains the human subjects, overview of experimental system, method to measure hand griping patterns, algorithm to calculate heart rate using pressure sensor sheet. Section 3 describes the experiment results, which were crosschecked by gold standard measurement system. Limitation of this research and discussion are presented at Section 4. At last conclude this paper in Section 5.

\section{Human Subjects and Method}

\subsection{Human Subjects}

Six healthy young males (age: $29.5 \pm 5.7$ years old, height: 1.74 $\pm 0.04 \mathrm{~m}$, body mass index: $23.19 \pm 1.93 \mathrm{~kg} / \mathrm{m}^{2}$ ) volunteered for the experiment. No subject reported medical constrain, motion sickness, drug abuse and alcoholism.

After the authors explained the objectives of this study, informed consent was obtained from all volunteers before the experiments. All experiments were conducted in accordance with the Declaration of Helsinki. Experiment in this study was approved by the Ethics Committee of the Clinical Trial Centre, Department of Medical Innovation, Osaka University Hospital (No.305, August 21, 2014).

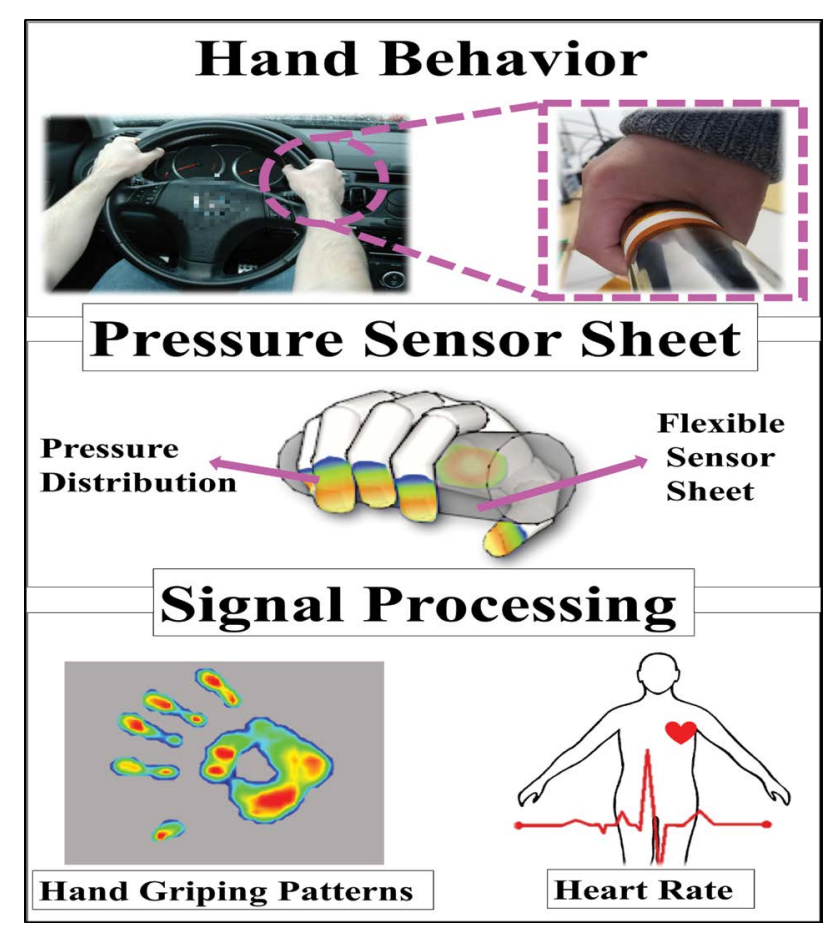

Figure 1: Concept of measuring hand griping patterns and heart rate from pressure sensor sheet.

\subsection{Experiment System}

Figure 2 shows the crosscheck-based experiment system utilized in our study. The system is consisted of two main parts: hand griping measurement system (Magenta Square at Figure 2) and gold standard measurement system (yellow square at Figure 2). The role of former system is to record the subtle pressure changing during hand griping. This part of system is made up of a flexible pressure sensor sheet, a therapy parallel bar $(\phi=42.70 \mathrm{~mm})$, a controller and computer. We roll up the pressure sensor sheet onto the therapy parallel bar and ask subjects to grasp the bar with six different griping patterns and steer still for 60 seconds, every volunteer perform tests for five times. Controller plays the role in transferring measured pressure data to computer. Data are analysed using MATLAB ${ }^{\mathrm{TM}}$ and details about signal processing will be performed later in this paper.

Overview of pressure sensor sheet utilized in our study is drawn at Figure 3. Figure 3a shows an example of measured pressure distribution when pressing the sensor, red areas represent part that had high pressure value while green parts indicate lower value. The pressure sensor sheet is made by organic flexible thin-film transistors (TFT). TFT has attracted considerable attention in next generation electronic application such as flexible displays, as well as they can be fabricated on plastic film using a solution process such as a printing method. Due to this feature, this pressure sensor sheet can be rolled to cylinder or bundled to steering wheel. The distance between each TFT is $4 \mathrm{~mm}$ at vertical direction and $5 \mathrm{~mm}$ at horizontal direction, the maximum sampling frequency for the pressure sensor sheet is up to $200 \mathrm{~Hz}$. The 


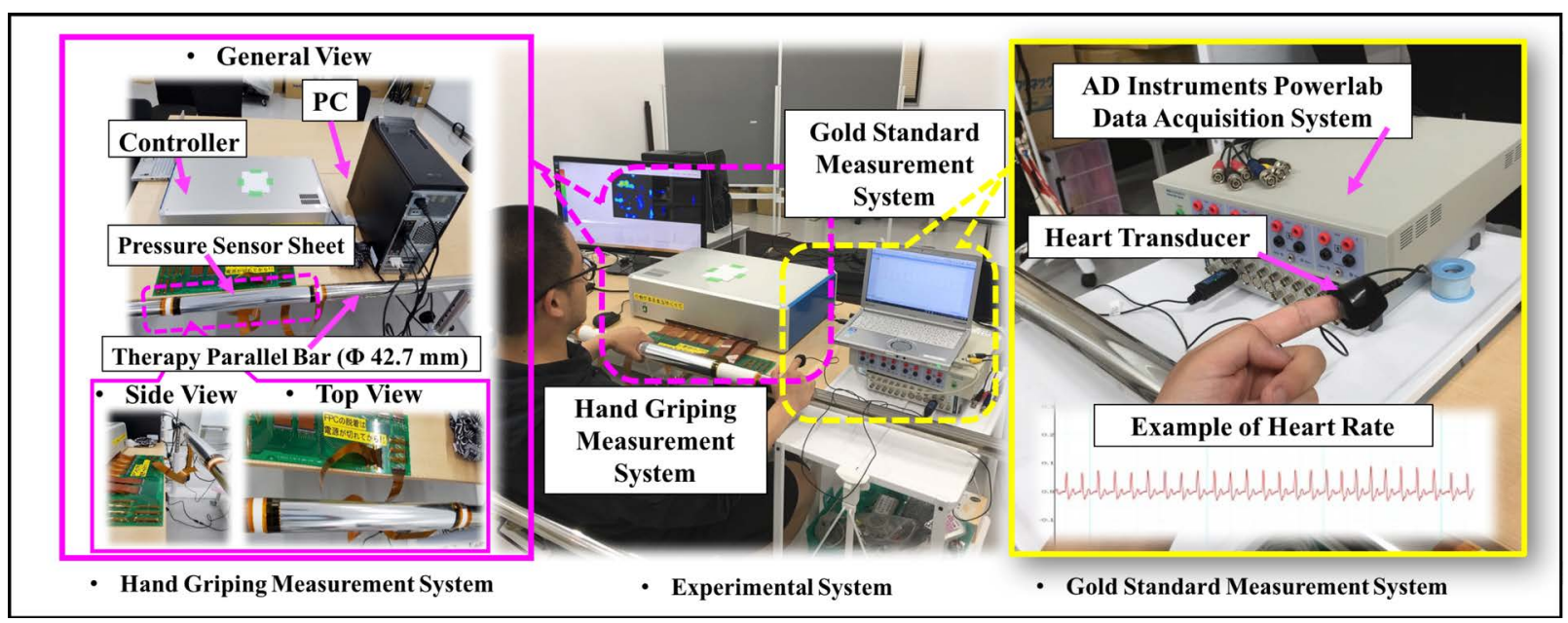

Figure 2: Overview of experiment system.

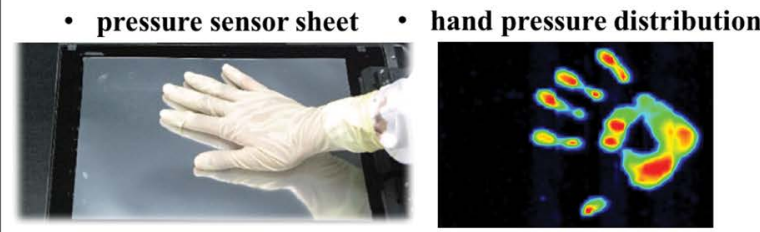

(a) overview of pressure sensor sheet

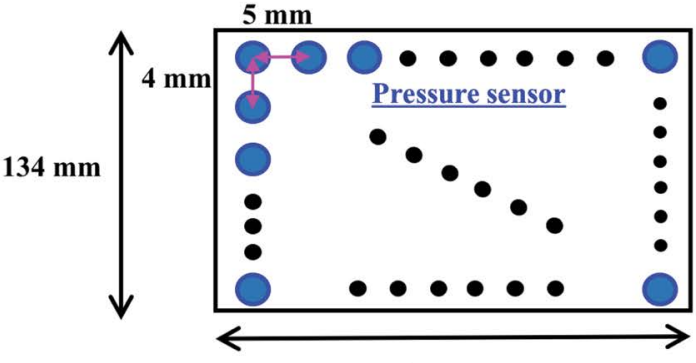

$375 \mathrm{~mm}$

(b) size and pressure sensors arrangement

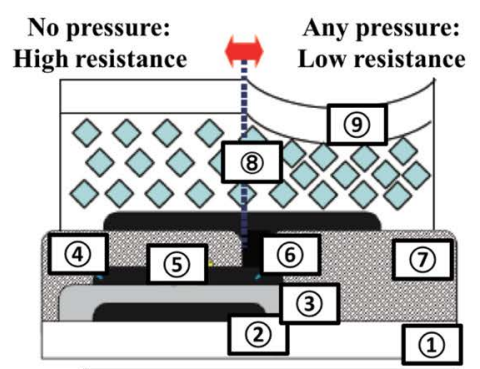

(1) Polyimide (substrate)

(2) Al (gate)

(3) $\mathrm{AlO}_{\mathrm{x}}$ (dielectric)

(4) Au (source \& drain)

(5) Semiconductor

(6) Au (source \& drain)

(7) C14-SAM

(8) Crystal

(9) Transparent electrode

(c) construct of pressure sensor sheet

Figure 3: Overview of pressure sensor sheet.

pressure sensor sheet has the number of 2,700 $(34 \times 75)$ pressure 2.3 Calibration for Pressure Sensor Sheet

sensors than and as large as an A4 printing paper (Figures $3 \mathrm{~b}$ and $3 c$ shows the operation principle of pressure sensor sheet. Once there is pressure on the surface of sensor, resistance of electronic film decreases due to the squeezing of crystal. Then pressure signal is transformed into electronic signal and is displayed by different colour according to pressure amplitude.

Powerlab $^{\circ} \mathrm{AD}$ Instruments has been recognized as a reliable data acquisition system in wide research, we believe the reliable performance of Powerlab ${ }^{\circ}$ could meet our requirement as a gold standard system. As shown in yellow square at Figure 2, TN1012/ ST heart rate transducer (typical output: $100 \mathrm{mV}$, weight including cable: $50 \mathrm{~g}$, size of transducer: $23 \mathrm{~mm}$ diameter $\times 11 \mathrm{~mm}$ thick, cable length: $2.90 \mathrm{~m}$ ) is bundled at volunteers' left index fingers. Measured heart rate data are used to crosscheck our experiment results.
Instrument calibration, as a vital step before various types of experiments, is utilized to establish reference point so that instruments produce accurate and reliable experiment results. Before volunteers began the experiment, we firstly perform calibration test as drawn in Figure 4a. Briefly, calibration system contains three main parts: pressure sensor sheet, a digital force gauge and one piece of surgical training skin. A high performance digital force gauges (FGP20 ONIDEC-SHIMPO Co. Ltd., sampling frequency: $1000 \mathrm{~Hz}$, range: $\pm 200 \mathrm{~N}$, error: $\pm 0.2 \%$ ) can transfer data to computer through USB port and specialized software Toriemon. In order to maintain high calibration accuracy, surgical training skin LM-021A Opeskin (๑KOKEN Co. Ltd., size $9 \mathrm{~cm}$ diameter $\times 1.50 \mathrm{~cm}$ height, $60 \mathrm{~g}$ weight) was tied up on the pressure sensor sheet to represses realistic touch of skin and resistance as close as volunteers steer the parallel bar. 


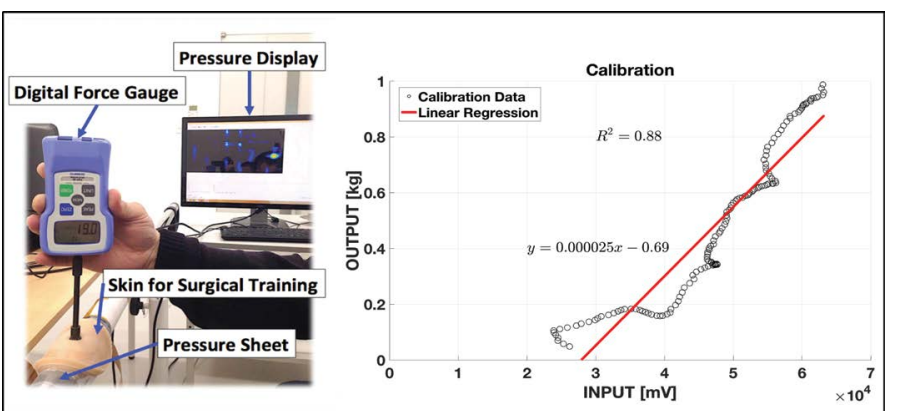

(a) Method of calibration

(b) Linear Regression for calibration

Figure 4: Overview of calibration for hand griping pressure data.

In this study, linear regression was calculated for calibration. Linear regression has been widely accepted as an approach for modeling the relationship between two variables [13]. We considered there was only one independent variable Xinput and response variable Youtput, simple linear regression can be written as:

$$
Y_{\text {output }}=\beta X_{\text {input }}+\varepsilon
$$

where $\beta$ is the slope and $\varepsilon$ is the intercept. In our calibration procedure, Xinput indicated data recorded from pressure sensor sheet and Youtput stood for force measured by digital gauge. The main purpose for linear regression is to find out the $\beta$ and $\varepsilon$. In order to find out a better fit, coefficient of determination (R2) needs to be calculated. $\mathrm{R} 2$ represents how good a linear regression model can predict the data, $\mathrm{R} 2$ ranges from 0 to 1 , the higher the amplitude is, and the better the model can predict the data. R2 can be calculated as following:

$$
R^{2}=1-\frac{\sum_{i=1}^{n}\left(y_{i}-\hat{y}\right)^{2}}{\sum_{i=1}^{n}\left(y_{i}-\bar{y}\right)^{2}}
$$

where $\bar{y}$ is the calculated value of Youtput and $\bar{y}$ represents the mean of Youtput.

Figure $4 \mathrm{~b}$ shows the results of linear regression utilized in our study. Finally, we fit independent variable Xinput to response variable Youtput when $\beta=0.00025, \varepsilon=-0.69$. It was considered that $\mathrm{R} 2$ is high enough $(\mathrm{R} 2=0.88)$ to indicate a strong connection between pressure sensor sheet and digital force gauge.

\subsection{Algorithm for Hand Griping Patterns and Heart Rate Calculation}

Figure 5 shows the algorithm for calculating hand griping from pressure sensor sheet. Figure $5 \mathrm{a}$ describes an image of hand griping pressure distribution in particular time. Figures $5 b$ and $5 c$ shows matrix of pressure sensors and pressure signals, pressure sensor sheet data (PSS) are made of row $34 \times$ column 75 matrix, which contained all pressure data on the pressure sensor sheet.

Next step is to calculate the summation of pressure value (SP) in Figure $5 c$ using the following equation and an example is drawn at Figure 4d:

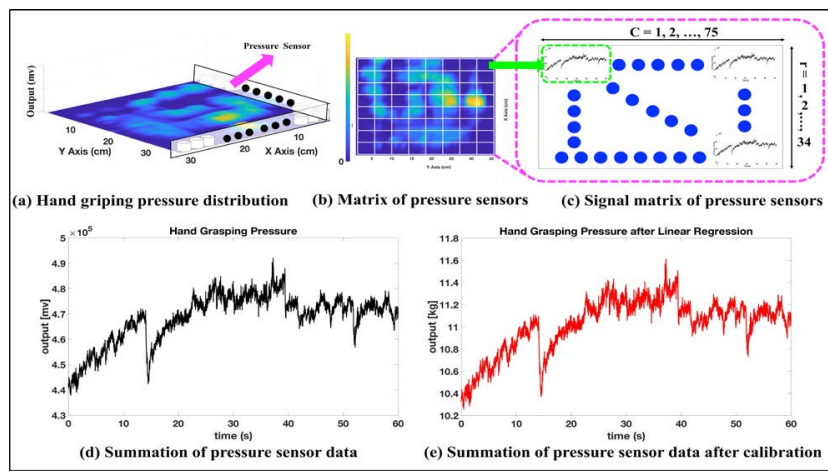

Figure 5: Algorithm of calculating hand griping pressure.

$$
S P=\sum_{i=1}^{t} \sum_{y=1}^{c} \sum_{x=1}^{r} P S S(t) . \text { matrix }(x, y)
$$

where $\mathrm{t}$ is the time, $\mathrm{r}$ and $\mathrm{c}$ represent the row and column respectively, $r=1,2 \ldots, 34, c=1,2 \ldots, 75$.

Figure $5 \mathrm{e}$ shows the results of hand griping pressure while subject holds still the therapy ball for $60 \mathrm{~s}$ after calibration aforementioned using Equation 1.

Data recorded from hand griping pressure are frequently intermingled with noise from electrical devices or human motion. Thus cutting off these signals is an essential processing before we acquire the heart rate data. It is well known that for healthy adult, maximum heartbeat per minute (HBPM) is from 60 to 100 HBPM [14], which means the frequency of heartbeat is between $1.0 \mathrm{~Hz}$ to $1.6 \mathrm{~Hz}(60 \mathrm{HBPM} / 60 \mathrm{~s}=1.0 \mathrm{~Hz}, 100 \mathrm{HBPM} / 60 \mathrm{~s} \approx 1.6 \mathrm{~Hz})$. As a consequence, we use band pass filter (BPF), $1.0 \mathrm{~Hz}$ low pass frequency and $1.6 \mathrm{~Hz}$ high pass frequency to cut off the raw data obtained from hand griping pressure. An example of hand griping pressure data processed after BPF in $20 \mathrm{~s}$ is drawn at Figure $6 \mathrm{a}$ in red curve. For a healthy adult, regular heartbeats make the red curve fluctuate regularly, thus we hypothesize that the numbers of local maximum points (red triangles at Figure $3 \mathrm{c}$ as the estimated heart beats (hb). Then measured value (MV) of heart rate (hr) can be calculated using the equation as following:

$$
h r=\frac{h b}{60 s}
$$

where hb is the number of local maximum points.

Actual value (AV) of heart rate measured from gold standard system is also calculated by Equation 4, and an example is drawn at Figure 6b. Blue curve indicates the ECG signal and blue triangles are the heart beats.

Then, it is necessary to evaluate the accuracy and agreement among MV through hand griping pressure and AV from gold stand measurement system. Firstly, accuracy rate (AR) can be calculated using following equation:

$$
A R(\%)=100 \%-\text { error } \%=100 \%-\frac{|A V-M V|}{A V} \times 100 \%
$$

In order to identify the relationship between proposed method and gold standard measurement system, we calculate the linear regression between AV and MV. Meanwhile, we 


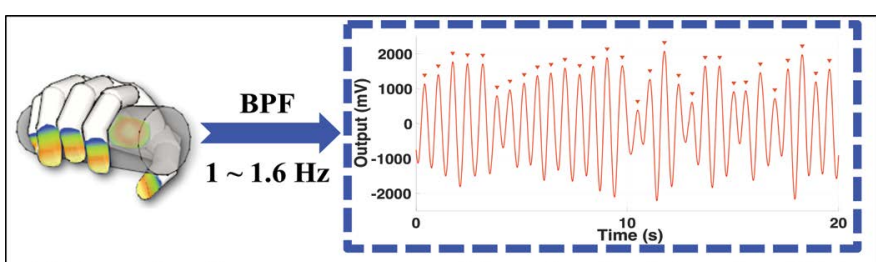

(a) Example of heart rate measured from hand griping after BPF

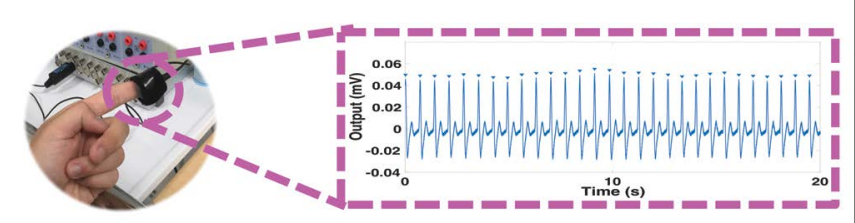

(b) Example of heart rate measured from gold standard

Figure 6: Examples of heart rates measured from hand griping (a) and gold standard measurement (b). BPF: band pass filter. Examples are shown in $20 \mathrm{~s}$.

also evaluate the agreement among MV and AV using BlandAltman plot. The Bland-Altman plot is formed by plotting the differences $d$ on the vertical axis while the averages $(\mathrm{AV}+\mathrm{MV}) / 2$ on the horizontal axis. $\mathrm{d}$ can be formed as:

$$
d=A V-M V
$$

At the Bland-Altman plot, a horizontal line representing the bias is drawn at $\bar{d}$, which is measured by the mean of the difference $\mathrm{d}$ using the following equation:

$$
\bar{d}=\frac{1}{n} \sum_{i=1}^{n} d_{i}
$$

where $\bar{d}$ represents the bias, $\mathrm{d}$ represents the difference, and $\mathrm{n}$ represents the number of sample data, $n=1,2 \ldots, 30$.

Usually, there are two more additional horizontal lines known as limits of agreement, which can be calculated as $\bar{d} \pm 1.96 S_{d}$, where 1.96 stands for the $\mathrm{z}$-value used to form $95 \%$ limits for a unit-normal random variable, $S_{d}$ is the standard deviation.

\section{Results}

Figure 7 shows the visualization results of six different hand griping patterns. Pictures on the left side are filmed by digital camera in order to check the results, red area at hand model pictures shows the engaged parts of hand. The 3-D plots shows the hand griping pattern and hand griping strength was calculated through linear regression. Colour bar indicates the hand pressure variation. Light yellow stands for high magnitude and dark blue represents low value.

From Figure 7a, it can be observed that when subject steered Altman Analysis. For Figure 8a, Heart rates measured from parallel bar only with their thumb, index and middle fingers, pressure sensor sheet when subjects perform hand griping there were only three yellow areas that represents three fingers (MV) showed strong connection between the heart rate while the rest part remained blue, which means in these areas, measured from gold standard (AV), R2 $=0.866$. Figure 8b pressure closed to zero. At this moment, hand griping strength indicates Bland-Altman Analysis results, black line in the was less than $6 \mathrm{~kg}$. From Figures $7 \mathrm{~b}-7 \mathrm{e}$, with increase numbers of middle of Figure $8 \mathrm{~b}$ represents the $\bar{d}$, which is calculated engaged fingers and using the whole palm to steer the bar, hand by Equation 7, and two red lines represent the limits of griping strength rose from $9.30 \mathrm{~kg}$ to $17.60 \mathrm{~kg}$, also yellow area agreement. It is clear that most points are distributed at the took huge part of the 3-D plot. It can be also observed that yellow area between two red lines. Heart rate measurement through 
Table 1: Results of heart rate measured.

\begin{tabular}{ccccc}
\hline Subjects & $\begin{array}{c}\text { Measured Value }(\mathbf{b p m}) \\
\text { mean } \pm \mathbf{S}_{\mathbf{d}}\end{array}$ & $\begin{array}{c}\text { Actual Value(bpm) } \\
\text { mean } \pm \mathbf{S}_{\mathbf{d}}\end{array}$ & $\begin{array}{c}\text { Accuracy Rate }(\%) \\
\text { mean } \pm \mathbf{S}_{\mathbf{d}}\end{array}$ & \multicolumn{2}{c}{ p-Value } \\
\hline 1 & $84.8 \pm 0.5$ & $85.8 \pm 1.8$ & $97.9 \pm 0.9$ & 0.68 \\
2 & $84.8 \pm 2.1$ & $85.2 \pm 2.3$ & $99.6 \pm 0.6$ & 0.93 \\
3 & $79.6 \pm 4.5$ & $80.4 \pm 4.2$ & $99.0 \pm 0.6$ & 0.9 \\
4 & $74.2 \pm 2.4$ & $70.2 \pm 3.0$ & $94.1 \pm 5.7$ & 0.78 \\
5 & $78.8 \pm 2.1$ & $81.4 \pm 2.9$ & $96.9 \pm 2.2$ & 0.68 \\
6 & $69.6 \pm 2.1$ & $66.6 \pm 1.8$ & $94.8 \pm 4.0$ & 0.8 \\
\hline
\end{tabular}

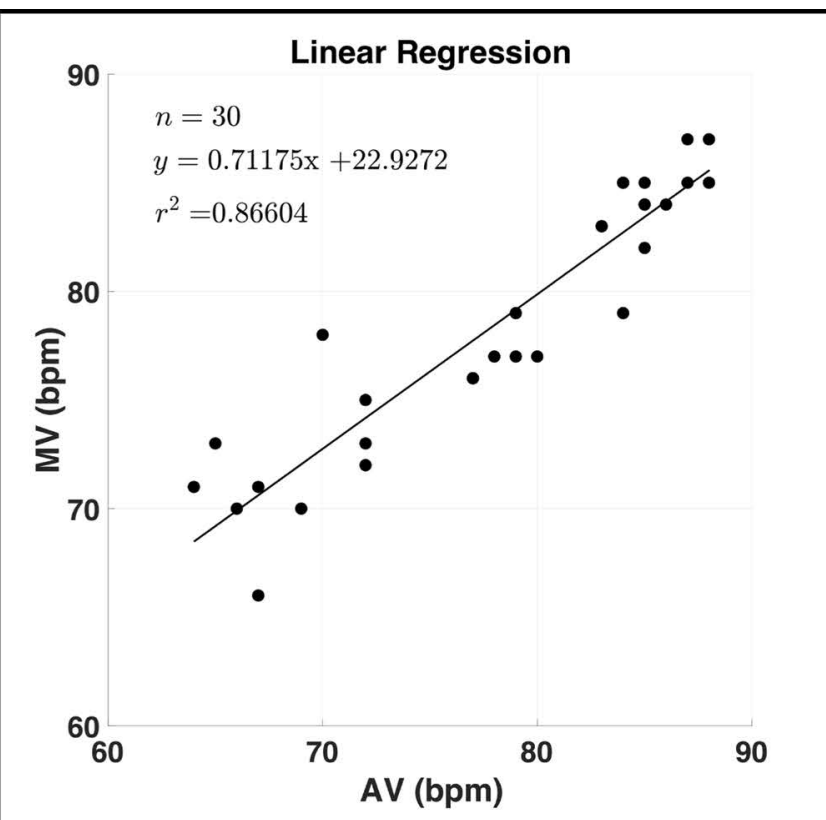

(a) Results of linear regression

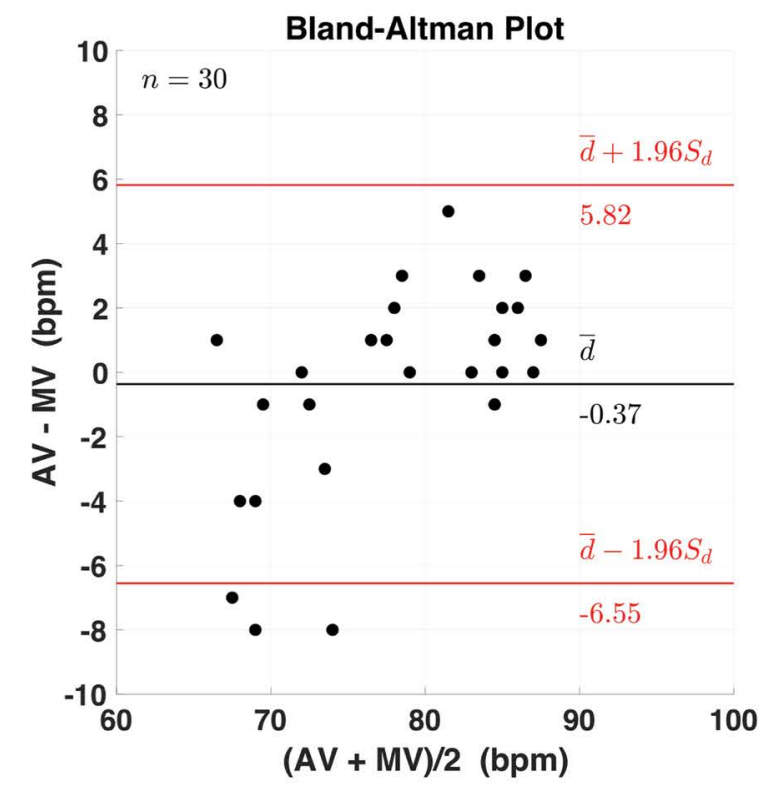

(b) Results of Bland-Altman Analysis

Figure 8: Results of relationship between heart rates from hand griping $(M V)$ and gold standard measurement $(A V)$. proposed method had $95 \%$ of difference fall within $-6.55 \mathrm{bpm}$ and $\pm 5.82 \mathrm{bpm}$. It is also noted that of the 30 measurements, $10 \%(n=3)$ are outside the confidence interval of $95 \%$.

\section{Discussion}

To our best knowledge, our study for the first time proposed a method to measure hand griping patterns and heart rate simultaneously through pressure sensor sheet. Experiment results showed high accuracy rate and good agreement in detecting heart rate without any uncomfortable electrodes or cables on subjects. Nevertheless, there are still some limitations that should be improved in future work.

In this basic study, we only asked volunteers to hold the parallel bar instead of a real steering wheel and no subjects reported sleepy during experiments, thus there lacked a real driving environment and heart rate when subjects feel drowsy. It is necessary to emphasize that experiment for real driving with drowsiness is an extremely highly risky task even though there is sufficient protection and test road. Considering the potential risk and high cost for real experiment, simulation system should be taken into consideration in the future study. Jibo et al. used Hyper Drive Authoring SuiteTM and Drive Safety's Vection Simulation SoftwareTM to test their wearable devices [15]. These systems can provide a low-cost but high-resolution meanwhile real time simulator for both clinical and research area. Thus as future work, we intend to upgrade our experiment in order to create an immersive test experience. Also, advanced pressure sensor that can perfectly be rolled on the steering wheel is necessary when performing real driving test.

It is well known that time series heart rate data can also be utilized to calculate HRV, the variations of beat to beat intervals in the heart rate [16]. The ration of high frequency band (HF, 0.15 0.40 Hz, is influenced by parasympathetic activity) and low frequency band (LF, $0.04 \sim 0.15 \mathrm{~Hz}$, is influenced by sympathetic and parasympathetic activities), which is known as LF/HF ratio was found decreased when subjects changed from waking state into sleepy state [17, 18]. Coincidentally, research indicating the relationship between four different conditions and HF/LF power showed that drowsiness resulted in higher HF power and low LF 
power, when people were awake; they presented lower HF References and LF power $[19,20]$. However, ECG in previous studies was depended on original biomedical monitor. ECG equipment needs electrodes and cables that would make subjects uncomfortable as well as complicate experiment setting. Thus, as future work in our study, HRV analysis will be performed to verify human spiritual condition through hand griping in order to take a closer inspection about driver's behaviours at steering wheel.

\section{Conclusion}

In this paper, we proposed a cableless measurement for measuring hand griping patterns and heart rate simultaneously through flexible pressure sensor sheet. Hand griping patterns were measured from hand pressure distribution and heart rates were measured from hand griping pressure value filtered with band pass filter. Experiment results were crosschecked by gold standard measurement system. According to experiment results, it was found that proposed method can detect heart rate with high accuracy (94\%) and good enough agreement compared with gold standard system using linear regression and Bland-Altman analysis.

As the limitation in present research, only six healthy males participated in the experiment and no volunteer reported they felt drowsy during the tests. We only asked volunteers to hold the parallel bar instead of a real steering wheel, there lacked heart rate when subjects feel drowsy and a real driving environment. Thus as future work, measuring biological signals when subjects feel sleepy is necessary and we also intend to upgrade our experiment in order to create an immersive test experience.

\section{Acknowledgements}

This study was funded by a Hirose International Scholarship Foundation (Grant No. J770703161), and was a part of a joint research with KONOIKE Transport Co, Ltd. We appreciated the technology support from Dai Nippon Printing Co. Ltd.

We acknowledge all members of our laboratory. Special thanks go to Yukari TANIYAMA for her support during the paper work.

\section{Author Contribution}

All authors have given approval of the final version to be published. T. Wang and H. Jeong have drafted the article; T. Wang and $\mathrm{H}$. Jeong have contributed to the conception and experiment design; T. Wang, H. Jeong, A. Guo, and T. Ohno collected and managed experiment data; T. Wang, $H$. Jeong performed data analysis, results and discussion; T. Ohno, N. Li, and Y. Ohno revised the article.

\section{Competing Interests}

The authors declare no conflicts of interest associated with this manuscript.

1. WHO. Global Status Report on Road Safety. 2015; 1-12.

2. Kline TJ. Using Efficient Steering Techniques. J Traffic Safety Educ. 2007; 55: 14-16.

3. https://www.gov.uk/guidance/the-highway-code

4. Schiro J, Gabrielli F, Pudlo P, Djemai M, Barbier F. Steering Wheel Hand Position in Low-Speed Maneuvers. Transp Res Part F Traffic Psychol Behav. 2013; 21: 133-45.

5. Altobelli A. Human Hand Motor Control Studies BT. Haptic Devices for Studies on Human Grasp and Rehabilitation. In: Altobelli A, editor. Springer Series on Touch and Haptic Systems Cham: Springer International Publishing; 2016. p. 7-17.

6. Tefft BC. The Prevalence and Impact of Drowsy Driving. 2010 .

7. McCartt AT, Rohrbaugh JW, Hammer MC, Fuller SZ. Factors Associated With Falling Asleep At The Wheel Among Long-Distance Truck Drivers. Accid Anal Prev. 2000; 32: 493-504.

8. Ueno $\mathrm{H}$, Kaneda $\mathrm{M}$, Tsukino $\mathrm{M}$. Development of Drowsiness Detection System. Proceedings of the Vehicle Navigation and Information Systems Conference; 1994 Aug 15-20; Yokohama, Japan: IEEE; 1994.

9. Lyznicki JM, Doege TC, Davis RM. Sleepiness, Driving, and Motor Vehicle Crashes. JAMA. 1998; 279: 1908-1913.

10. Bourgeois-Bougrine S, Carbon P, Gounelle C, Mollard R, Coblentz A. Perceived Fatigue for Short- and LongHaul Flights: A Survey Of 739 Airline Pilots. Aviat Space Environ Med. 2003; 74: 1072-1077.

11. Brown ID. Driver Fatigue. Hum Factors. 1994; 36: 298314.

12. Clapers JG, Casanella R. A Fast And Easy-To-Use Ecg Acquisition And Heart Rate Monitoring System Using A Wireless Steering Wheel. IEEE Sensor Journal. 2012; 12: 610-616.

13. Yu X. Real-Time Nonintrusive Detection Of Driver Drowsiness- Phase II. Technical Report for University of Minnesota. Center for Transportation Studies University of Minnesota. 2009.

14. Freedman DA. The Regression Line. In: Statistical Model: Theory and Practice. Cambridge: Cambridge University Press; 2009. p. 18-28.

15. https:/healthyforgood.heart.org/move-more/articles/ target-heart-rates. 
16. He J, Choi W, McCarley JS, Chaparro BS, Wang C. Texting 19. Tsunoda M, Endo T, Hashimoto S, Honma S, Honma KI. while driving using Google Glass ${ }^{\mathrm{TM}}$ : Promising but not distraction-free. Accid Anal Prev. 2015; 81:218-229. Effects of light and sleep stages on heart rate variability in humans. Psychiatry Clin Neurosci. 2001; 55:285-286.

17. Malik M. Guidelines Heart rate variability. Eur Heart J. 1996; 20. Vicente J, Laguna P, Bartra A, Bailón R. Drowsiness detection $17: 354-381$. using heart rate variability. Med Biol Eng Comput. 2016;

18. Carl JS, William CO, Terence MD. Nightly Variability of Sleep54:927-937. Disordered Breathing Measured over 3 Nights. Otolaryngol Head Neck Surg. 2004; 131: 837-843. 